\title{
A Physical Model-based Correction for Charge Traps in the Hubble Space Telescope's Wide Field Camera 3 Near-IR Detector and Its Applications to Transiting Exoplanets and Brown Dwarfs
}

\author{
Yifan Zhou ${ }^{1,4}$, Dániel Apai ${ }^{1,2,3}$, Ben W. P. Lew ${ }^{2}$, and Glenn Schneider ${ }^{1}$ \\ ${ }_{1}^{1}$ Department of Astronomy/Steward Observatory, The University of Arizona, 933 N. Cherry Avenue, Tucson, AZ 85721, USA; yzhou@as.arizona.edu \\ ${ }^{2}$ Department of Planetary Science/Lunar and Planetary Laboratory, The University of Arizona, 1640 E. University Boulevard, Tucson, AZ 85718, USA \\ ${ }^{3}$ Earths in Other Solar Systems Team, NASA Nexus for Exoplanet System Science, 933 N. Cherry Avenue, Tucson, AZ 85721, USA \\ Received 2016 November 18; revised 2017 February 17; accepted 2017 February 28; published 2017 May 4
}

\begin{abstract}
The Hubble Space Telescope Wide Field Camera 3 (WFC3) near-IR channel is extensively used in time-resolved observations, especially for transiting exoplanet spectroscopy as well as brown dwarf and directly imaged exoplanet rotational phase mapping. The ramp effect is the dominant source of systematics in the WFC3 for timeresolved observations, which limits its photometric precision. Current mitigation strategies are based on empirical fits and require additional orbits to help the telescope reach a thermal equilibrium. We show that the ramp-effect profiles can be explained and corrected with high fidelity using charge trapping theories. We also present a model for this process that can be used to predict and to correct charge trap systematics. Our model is based on a very small number of parameters that are intrinsic to the detector. We find that these parameters are very stable between the different data sets, and we provide best-fit values. Our model is tested with more than 120 orbits ( 40 visits) of WFC3 observations and is proved to be able to provide near photon noise limited corrections for observations made with both staring and scanning modes of transiting exoplanets as well as for starting-mode observations of brown dwarfs. After our model correction, the light curve of the first orbit in each visit has the same photometric precision as subsequent orbits, so data from the first orbit no longer need to be discarded. Near-IR arrays with the same physical characteristics (e.g., JWST/NIRCam) may also benefit from the extension of this model if similar systematic profiles are observed.
\end{abstract}

Key words: brown dwarfs - instrumentation: detectors - planets and satellites: atmospheres

\section{Introduction}

The Hubble Space Telescope Wide Field Camera 3 (HST WFC3) near-infrared (near-IR) channel, is one of the most powerful and most frequently used instruments for exoplanet atmosphere observations. With its exceptional sensitivity and high photometric stability, WFC3 plays an essential role in high-cadence time domain observations, including transit grism spectroscopy (e.g., Swain et al. 2013; Kreidberg et al. 2014) and rotational phase mapping (e.g., Buenzli et al. 2012; Apai et al. 2013; Lew et al. 2016; Zhou et al. 2016). However, instrumental systematics in its near-IR channel either related to the detector (for a summary, see Wakeford et al. 2016) or the grism geometry (for a detailed description and correction, see Varley et al. 2015) prevent the attainment of photon noise limited performance. The oftencalled "ramp effect" (e.g., Berta et al. 2012) is an approximately exponentially shaped signal in the time domain. It is the most significant systematic affecting photometric efficacy. Different empirical profiles of the ramp effect were identified in various HST time-resolved observation data sets (Figure 1). It is expected that other instruments that employ detectors with similar architectures, such as JWST/NIRCAM and NIRSPEC, may also suffer from similar systematics.

Great effort has been devoted to the calibration of the ramp effect. Until now, the most popular and successful method has been the "divide-out-of-transit" method (e.g., Berta et al. 2012). This method uses an exponential/polynomial function (empirically derived from the out-of-transit parts of the light curves) to

\footnotetext{
${ }^{4}$ NASA Earth and Space Science Fellow.
}

correct the ramp effect. Several of the most precise HST transit spectroscopic observations (e.g., Berta et al. 2012; Kreidberg et al. 2014) have adopted this correction. However, this method has three important limitations. First, the ramp effect is significantly more severe in the first HST orbit than in the rest of the observations in sequential target visibility periods, ${ }^{5}$ and the first orbit cannot be corrected by the divide-out-of-transit method. Although the difference in systematics between the different orbits is not well understood, it is widely assumed that the telescope needs to thermally settle, i.e., to reach some sort of equilibrium. Therefore, the data from the first orbit of each visit are always excluded from data analysis, which results in the loss of approximately 100-200 orbits up to the end of HST cycle 24 . Second, the out-of-transit method requires a flat photometric baseline to fit the empirical functions that are used for correction. When intrinsic variability is present, such as in hot-Jupiter orbital phase curves and in rotational phase mapping observations of brown dwarfs and directly imaged exoplanets, this method is not reliable. Third, this method is not based on an understanding of the underlying physical mechanisms of the systematics. The ad hoc nature of this method makes it difficult to evaluate the applicability of this instrumental systematic when observations with different detector readout modes and illumination levels are obtained with different instrument configurations. A physically motivated ramp-effect model that enables the retention of all photometric data with post-processing (ramp calibration) is therefore highly desired.

\footnotetext{
5 During each orbit of HST ( 93 minutes) a typical target is visible for 40-54 minutes, depending on its celestial position. Therefore, HST only acquires science exposures during a fraction of each period, i.e., in the visibility period.
} 

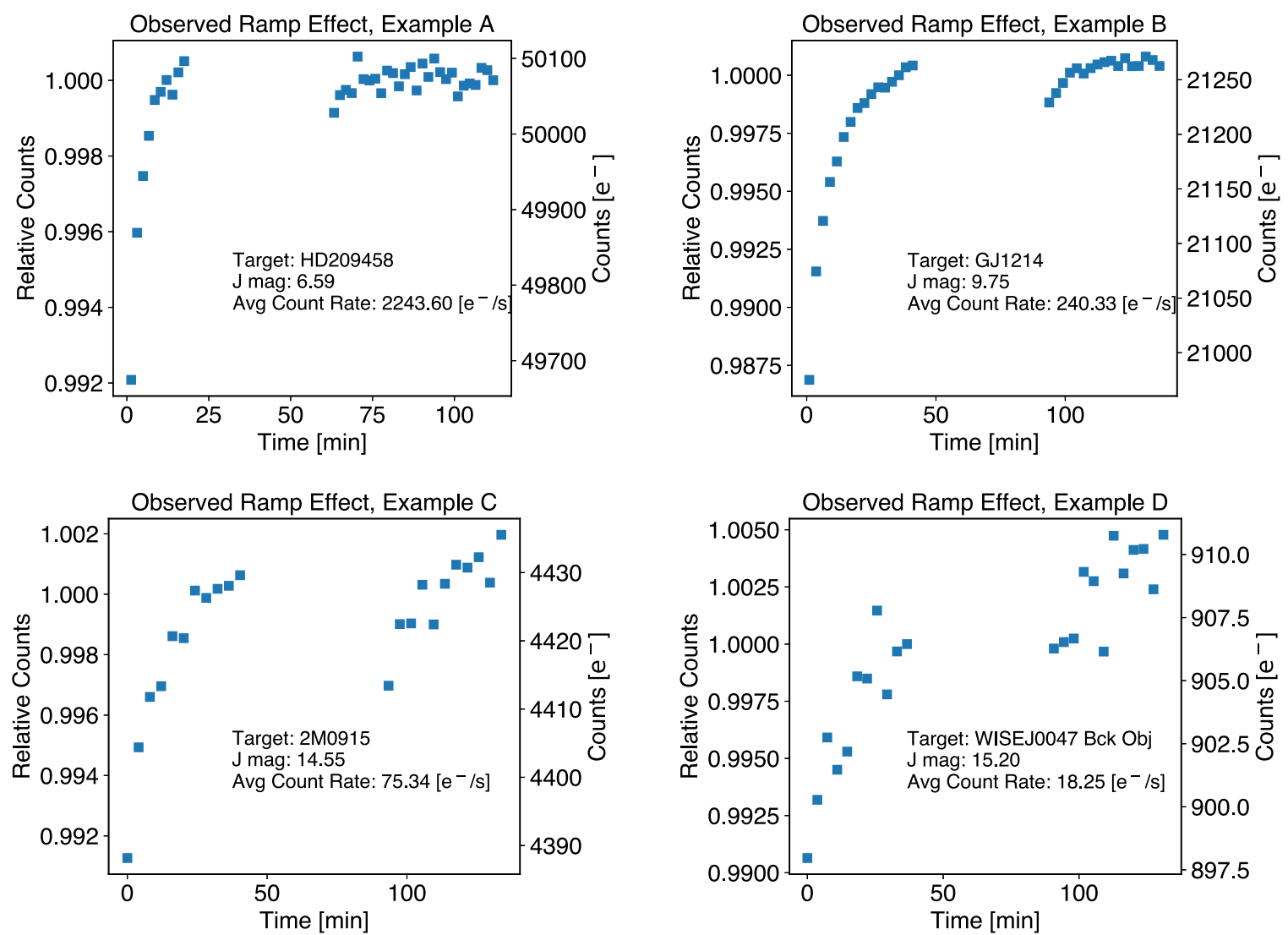

Figure 1. Different manifestations of the ramp effect are presented in various observations. With different target brightness, exposure times, and exposure sequences, the ramps have different shapes.

Charge trapping has been suggested to be the major cause of the ramp effect for infrared detectors. Agol et al. (2010) used electron trapping to attempt to explain the ramp effect of Spitzer's IRAC detectors, and they empirically described this effect with two exponential functions. Furthermore, in WFC3 data, the amplitudes of the ramp were found to be related to the exposure count levels (e.g., Berta et al. 2012), a finding that is broadly consistent with charge trapping.

Instead of using empirically estimated exponential functions to calibrate electron trapping, in this paper, we developed a physical model Ramp Effect Charge Trapping Eliminator (RECTE), which is described by the numbers of charge carrier traps, the trapping efficiency and the trap lifetimes in every pixel on the detector. We show that this model works extremely well with time-resolved observations taken in both scanning and staring modes. We explain the details of the model (Section 2), describe the model results (Section 3), demonstrate two examples of model applications (Section 3.1), and discuss the correction results, future observation planning suggestions, and model extensions for future instruments (Section 4) in the rest of the paper.

\section{The Ramp Effect Model}

\subsection{Physical Background}

The WFC 3 infrared channel detector is a $1 \mathrm{k} \times 1 \mathrm{k} \mathrm{HgCdTe}$ array (Dressel 2016). The detector was manufactured by Teledyne Scientific \& Imaging, and has an architecture of hybrid $\mathrm{HgCdTe}$ device grown on a CdZnTe substrate and indium-bonded to a Hawaii-1R MUX (Baggett et al. 2008).
The basic elements of the detector array are photodiodes. Photons are absorbed by the diodes to produce free charge carriers. The free charge carriers travel through the diodes following the electric potential and reach the depletion region of the diodes. The electric field maintained by the contact potential drives the charge carriers across the P-N junction, and the collected charge carries change the circuit gate voltage that are measured as the signal (Rieke 2012).

A highly repeatable characteristic of this type of detector is that the $\mathrm{P}-\mathrm{N}$ junction is de-biased as the signal is accumulated. This can change the response of the detector during a series of exposures (Rieke 2012) and is suggested to be the primary source of image persistence (Smith et al. 2008a). The latter authors proposed that charge traps in the depletion region of the $\mathrm{P}-\mathrm{N}$ junction are responsible for the image persistence, and based on this assumption established a qualitative theoretical framework to explain the image persistence phenomenon. They also suggest that charge carriers can be trapped as they diffuse across the depletion region. The trapping of the charge carriers lowers the level of the signal measured in the individual exposures. At the end of the exposure, the original width of the depletion is restored by reset, but the trapped charge carriers remain in the depletion region. In subsequent frames, the trapped charge carriers are released and diffuse back to the undepleted region, which generates a signal. This signal is manifested either as image persistence in the dark frame, or as a ramp-shaped light curve in exposure series.

Smith et al. (2008b) applied this model to calibrate the image persistence in the HgCdTe array of the SuperNova Acceleration Probe. They found that the integrated persistence profiles 

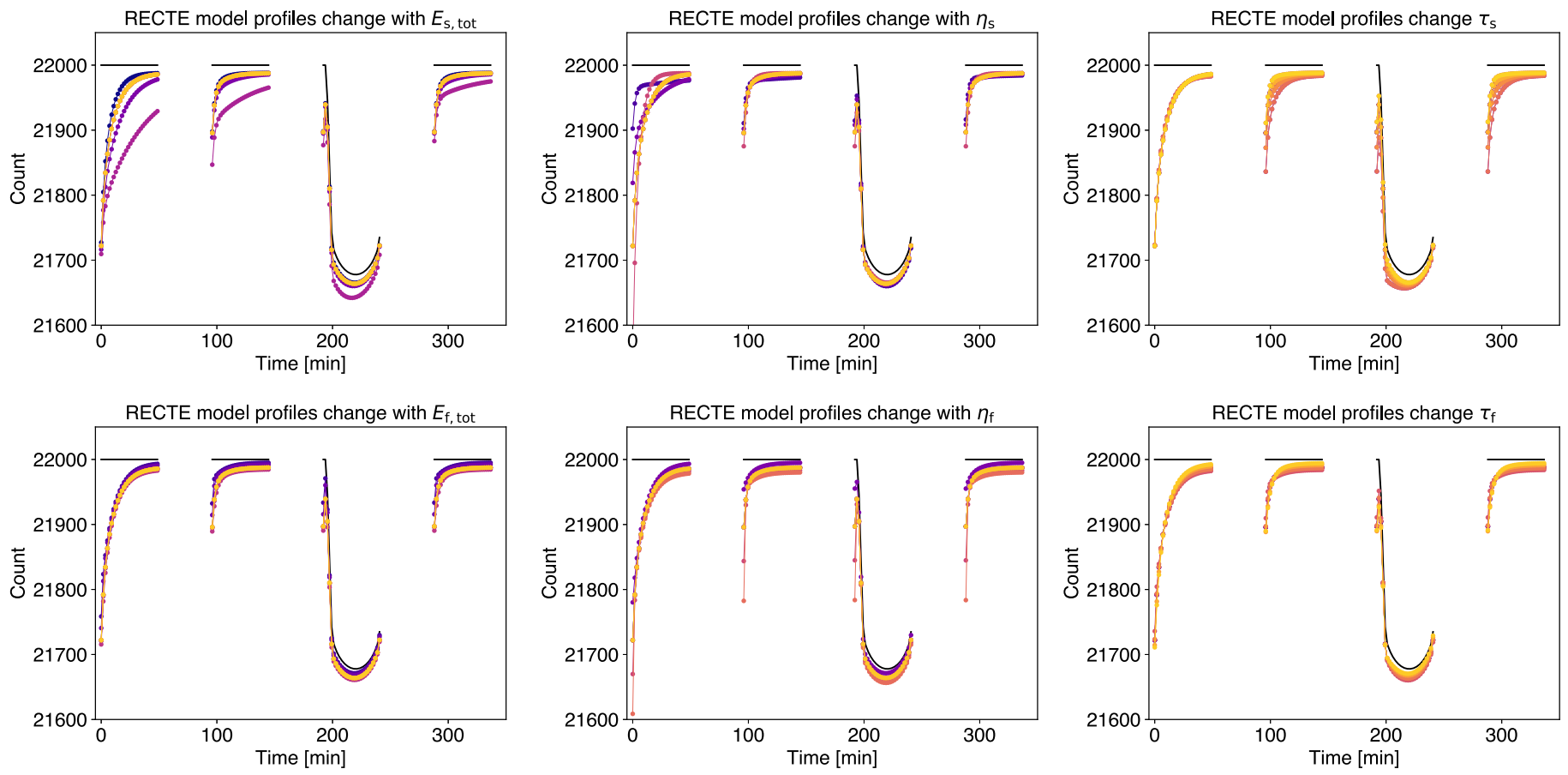

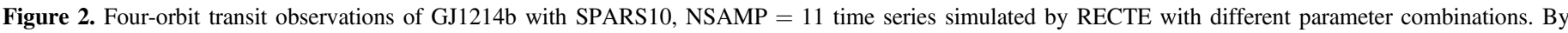

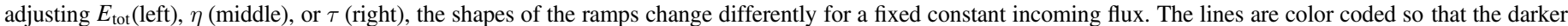
the line, the larger the changing model parameter is. The black lines are the light curves with no ramp effect.

can be calibrated using a double-exponential profile, of which the time constants of two exponential functions differed by more than an order of magnitude. They suggested that the two trap populations could be the result of the two types of charge carriers, electrons and holes, behaving differently. Furthermore, they found that the trapping efficiency has relatively small spatial variation, which provided evidence that individual pixels had similar trapping properties. Studies (e.g., Anderson et al. 2014; Long et al. 2015a) have explored the charge carrier trap properties of the WFC3 near-IR detector and detectors that share similar physical architectures. Long et al. (2015b) created a model for the persistence of WFC3 near-IR detector. These studies focused on the persistence behavior of the detector with a timescale of several minutes after high count level exposures. These studies aimed to describe the effects of charge trapping on the data by fitting empirical functions to the observed trends, an approach that only works imperfectly and is only applicable within sets of self-similar observations. The model we introduce here invokes the same physically motivated conceptual picture as previous studies (e.g., Smith et al. 2008a); however, our approach differs from the past qualitative and quantitative models in two important aspects.

First, our model assumes that charge trapping occurs immediately after the photons are absorbed and not later, i.e., charge trapping occurs only when the detector is illuminated. This difference results in a different behavior of the detector, which we demonstrate is more consistent with the existing observations than the predictions of the traditional model that assumes continuing trapping even in the non-illuminated state of the detector.

Second, our model aims at correcting for the ramp effect and not the image persistence. The later effect is only important at very high fluence levels. Therefore, our model does not aim to incorporate a complete description of charge trapping and release mechanisms and the effects of nonlinearity in the extreme case of near-saturation levels. Comparisons of our model predictions to observations shows that our model effecively reproduces data sets taken at moderately high fluence levels but not those taken at close to saturation levels. This indicates that physical effects not considered in our model play important roles when the detector is driven to saturation.

\subsection{Model Descriptions}

Our model is based on the charge carrier trapping theory of Smith et al. (2008a). However, instead of fitting empirically derived exponential functions, our model enables us to quantitatively model the charge carrier trapping processes, so that we can precisely calibrate ramp-effect-impacted timeresolved observations made with WFC3. In order to quantitatively constrain the behavior of the WFC3 detector, we generalize the theory with the following assumptions.

1. The detector pixels have two populations of charge carrier traps: a slow trap population that releases trapped particles with a long trapping lifetime and a fast trap population that releases trapped particles with a short trapping lifetime. The total numbers of traps per pixel for the two population are $E_{\mathrm{s} \text {,tot }}$ and $E_{\mathrm{f} \text {,tot }}$. The power-law persistence trend seen in studies of Long et al. (2012, 2015a) suggests the possibility of a more complex nature of the traps. We focus on two trap population model in this work and reserve the possibility to extend the model to multiple trap populations, which may become important when the detector is illuminated at levels close to saturation.

2. Charge carriers stimulated by incoming photon fluxes can fill the two populations of traps with efficiencies of $\eta_{\mathrm{s}}$ 
Table 1

Parameter List

\begin{tabular}{|c|c|c|}
\hline Parameter & Unit & Description \\
\hline$E_{\mathrm{s}, \text { tot }}, E_{\mathrm{f}, \text { tot }}$ & count & Total numbers of slow and fast traps \\
\hline$\eta_{\mathrm{s}}, \eta_{\mathrm{f}}$ & $\cdots$ & Trapping efficiencies for slow and fast traps \\
\hline$\tau_{\mathrm{s}}, \tau_{\mathrm{f}}$ & second & Trap lifetimes for slow and fast traps \\
\hline$E_{\mathrm{s}, 0}, E_{\mathrm{f}, 0}$ & count & $\begin{array}{l}\text { Number of slow and fast traps occupied at the } \\
\text { beginning of the visit. }\end{array}$ \\
\hline$\Delta E_{\mathrm{s}}, \Delta E_{\mathrm{f}}$ & count & $\begin{array}{l}\text { Extra number of charge carriers captured by slow } \\
\text { and fast traps during the observation gaps. }\end{array}$ \\
\hline$f$ & count $\mathrm{s}^{-1}$ & Illumination flux \\
\hline
\end{tabular}

and $\eta_{\mathrm{f}}$. The states of the two populations' traps are independent. The numbers of the trapped charge carriers at time $t$ are $E_{\mathrm{s}}(t)$ and $E_{\mathrm{f}}(t)$. The charge carrier trapping rates are proportional to incoming flux $f(\mathrm{t})\left[\mathrm{e}^{-} / \mathrm{s}\right]$ and to the number of unfilled traps $\left(\bar{E}(t)=E_{\text {tot }}-E(t)\right)$. With these assumptions, the charge carrier trapping rate can be expressed as Equation (1). ${ }^{6,7}$

$$
\frac{d E(t)^{+}}{d t}=\eta f(t) \frac{E_{\mathrm{tot}}-E(t)}{E_{\mathrm{tot}}}
$$

3. The two populations of trapped charge particles have lifetimes of $\tau_{\mathrm{s}}$ and $\tau_{\mathrm{f}}$. Under no illumination, the number of trapped charges follows exponential decay:

$$
\begin{gathered}
E(t)=E\left(t_{0}\right) \exp \left(-\frac{t-t_{0}}{\tau}\right) \text { when } f=0 \\
\frac{d E(t)^{-}}{d t}=-\frac{E(t)}{\tau} .
\end{gathered}
$$

Combining Equations (1) and (3), the complete form for trap number change follows

$$
\frac{d E(t)}{d t}=\eta f(t) \frac{E_{\mathrm{tot}}-E(t)}{E_{\mathrm{tot}}}-\frac{E(t)}{\tau} .
$$

4. Because traps are filled during the exposures, the charge carrier trapping rates decrease. Therefore, the number of detected electrons per unit time increases, giving the characteristic "ramp" shape. When all traps are filled, or the charge carrier release rate is equal to the trapping rate, the detected flux will be equal to the true flux. In addition, a notable number of charge carriers is released between the target visibility periods, when the detector is not illuminated by the target. Therefore, the light curves usually rise up again at the beginning of each subsequent orbit (see Figure 1).

5. The number of occupied traps is not necessarily zero at the beginning of each visit because (1) previous observations may have illuminated the same pixels as current exposures; (2) the detector received flux before the first exposure, as WFC3 has no shutter for the detector, and is occassionally unintentionally illuminated during telescope slewing. To reflect these possibilities, we introduced parameters $E_{\mathrm{s}, 0}$ and $E_{\mathrm{f}, 0}$ that represent

\footnotetext{
6 No subscripts of $s$ and $f$ means the expressions work for both trap populations.

Superscript ${ }^{+}$denotes trap gaining processes, and ${ }^{-}$denotes trap releasing processes.
}

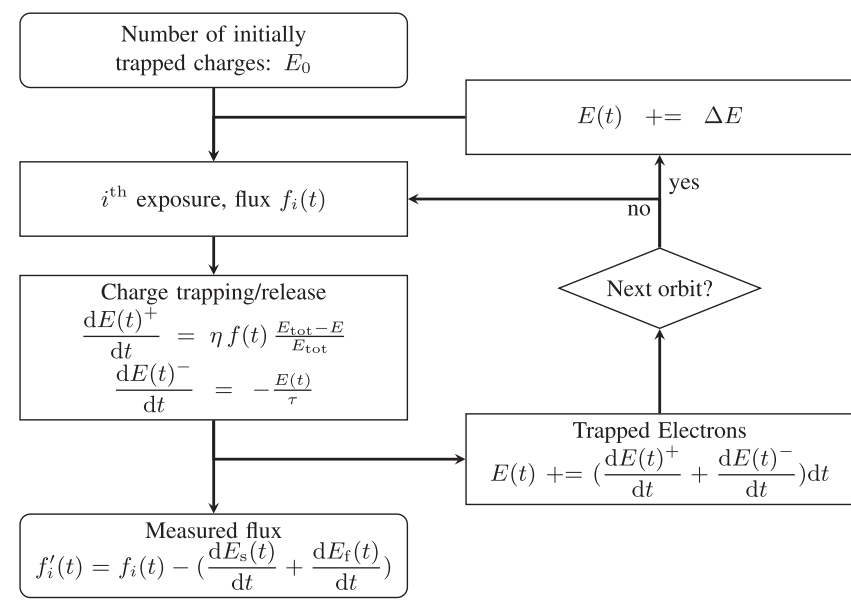

Figure 3. RECTE model processes presented with a flowchart.

the initially occupied number of traps. These parameters can vary from visit to visit, as well as from pixel to pixel.

6. Furthermore, because WFC3 may be illuminated by the target before the beginning of the science exposure series, parameters $\Delta E_{\mathrm{s}} \Delta E_{\mathrm{f}}$ are included to represent extra trapped charges during the time between visibility periods.

7. After evaluating a number of different data sets, we found no evidence for trapping parameters $\left(E_{\mathrm{tot}}, \eta\right.$, and $\left.\tau\right)$ to change with time; therefore, we assume that these parameters are intrinsic to the detector and constant with time. $E_{0}$ and $\Delta E$ can be different for different observations.

Table 1 summarizes the key parameters of our model and their physical meaning. We show the RECTE model profiles with different sets of model parameters in Figure 2.

The model is presented as a flowchart in Figure 3:

\subsection{Mathematical Solutions for Ramp Profiles}

From Equations (1) and (3), we can express $E(t)$ as a differential equation:

$$
\frac{d E}{d t}=\frac{d E^{+}}{d t}+\frac{d E^{-}}{d t}=\eta f(t)-\left(\frac{\eta f(t)}{E_{\mathrm{tot}}}+\frac{1}{\tau}\right) E
$$

The formal solution of (5) is

$$
E(t)=\left[\int \eta f(t) e^{\int\left(\frac{\eta f(t)}{E_{\mathrm{tot}}}+\frac{1}{\tau}\right) d t} d t+C\right] e^{-\int\left(\frac{\eta f(t)}{E_{\mathrm{tot}}}+\frac{1}{\tau}\right) d t} .
$$

We note that $E_{\mathrm{s}}(t)$ and $E_{\mathrm{f}}(t)$ are calculated separately using Equation (6) because we assume that the states of the two populations are independent.

Therefore, the measured flux is

$$
f^{\prime}(t)=f(t)-\frac{d E_{\mathrm{s}}(t)}{d t}-\frac{d E_{\mathrm{f}}(t)}{d t} .
$$

If the incoming photon flux is constant, Equation (5) has an analytical solution of

$$
E(t)=\frac{\eta f}{\frac{\eta f}{E_{\mathrm{tot}}}+\frac{1}{\tau}}+\left(E_{0}-\frac{\eta f}{\frac{\eta f}{E_{\mathrm{tot}}}+\frac{1}{\tau}}\right) e^{-\left(\frac{\eta f}{E_{\mathrm{tot}}}+\frac{1}{\tau}\right) t}
$$


where $E(t=0)=E_{0}$. The observed flux is the intrinsic flux subtracted by the charge carriers that are trapped:

$$
\begin{aligned}
f^{\prime}(t)= & f-\frac{d E_{\mathrm{s}}(t)}{d t}-\frac{d E_{\mathrm{f}}(t)}{d t} \\
= & f-\left[\eta_{\mathrm{s}} f-E_{\mathrm{s}, 0}(t)\left(\frac{\eta_{\mathrm{s}} f}{E_{\mathrm{s}, \text { tot }}}+\frac{1}{\tau_{\mathrm{s}}}\right)\right] e^{-\left(\frac{\eta_{\mathrm{s}} f}{E_{\mathrm{s}, \mathrm{tot}}}+\frac{1}{\tau_{\mathrm{s}}}\right) t} \\
& -\left[\eta_{\mathrm{f}} f-E_{\mathrm{f}, 0}(t)\left(\frac{\eta_{\mathrm{f}} f}{E_{\mathrm{f}, \text { tot }}}+\frac{1}{\tau_{\mathrm{f}}}\right)\right] e^{-\left(\frac{\eta_{\mathrm{f}} f}{E_{\mathrm{f}, \mathrm{tot}}}+\frac{1}{\tau_{\mathrm{f}}}\right) t} .
\end{aligned}
$$

In the following, we discuss a few manifestations of our model.

1. At the beginning of the observations and in the case where $E_{0}$ is close to zero, the measured flux is $f^{\prime}(t)=\left(1-\eta_{\mathrm{s}}-\eta_{\mathrm{f}}\right) f$. Therefore, the amplitude of the ramp is mainly controlled by $\eta_{\mathrm{s}}+\eta_{\mathrm{f}}$.

2. When the number of filled traps reaches

$$
E=\frac{\eta f}{\frac{\eta f}{E_{\mathrm{tot}}}+\frac{1}{\tau}}
$$

where released electrons and trapped electrons are in equilibrium. In this case, a constant incoming flux results in a flat measured light curve.

3. If the irradiated count rates are low, $\left(\frac{\eta f}{E_{\mathrm{tot}}}+\frac{1}{\tau}\right) \ll 1$, the measured flux will be

$$
\begin{aligned}
f^{\prime}(t)= & \left(1-\eta_{\mathrm{s}}-\eta_{\mathrm{f}}\right) f \\
& +\left(\frac{\eta_{\mathrm{s}}^{2} f^{2}}{E_{\mathrm{stot}}}+\frac{\eta_{\mathrm{s}} f}{\tau_{\mathrm{s}}}+\frac{\eta_{\mathrm{f}}^{2} f^{2}}{E_{\mathrm{f}, \mathrm{tot}}}+\frac{\eta_{\mathrm{f}} f}{\tau_{\mathrm{f}}}\right) t .
\end{aligned}
$$

In this case, the ramp will become a linear profile.

\subsection{Constraining the Model Parameters}

We constrain our model by primarily using the scanning mode observations of the transiting exoplanet GJ1214b (Kreidberg et al. 2014, HST program 13021). This data set includes 15 visits that were taken between 2012 September and 2013 August. Each of the 15 visits included 4 orbits G141 transiting spectroscopic observations to observe one transit of GJ1214b, and the transits all occurred within the third orbit of each visit observations. The first 5 visits were taken with onedirectional scanning and SPARS10, NSAMP $=13$ exposures, and the last 10 visits are taken with two-directional (round trip) scanning and SPARS10, NSAMP $=15$ exposures.

We used the first two-orbit observations of every visit to constrain the model parameters, because the first two orbits had intrinsically flat light curves. The last two orbits in each visit were used to test the validity of the model. Kreidberg et al. (2014) discarded three visits for their analysis because of starspot crossing or guiding failure. The first two orbits of these visits were not affected by these problems. Therefore, we used all 15 visits to constrain the model parameters, but discarded the same three visits for model testing and validation.

The data reduction started with ima files produced by the STScI CalWFC3 pipeline that include all calibrated nondestructive readouts and followed the regular scanning mode data reduction routine described in Deming et al. (2013) and McCullough \& MacKenty (2012). We marked the pixels that

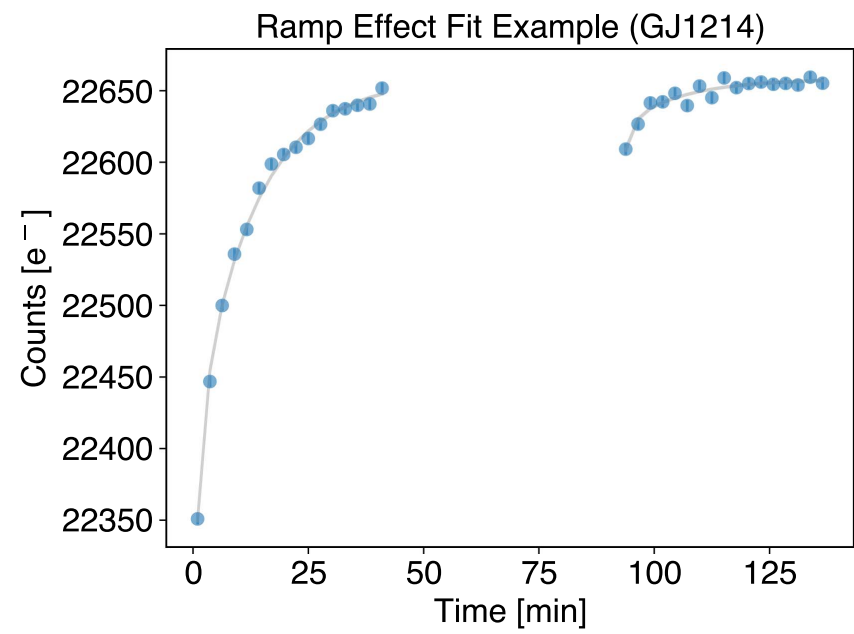

Figure 4. Example of the ramp-effect light curve fitted to the RECTE model curve. Blue points are observational data that come from the average of onepixel column of the spatial scan. The gray lines are the RECTE model curve fits, each of which is one randomly chosen realization of the MCMC chain. The two orbits are well fit by to the ramp model light curve with maximum likelihood.

have data quality flags of $4,16,32$, and 256 (bad pixel, hot pixel, unstable response, saturation) as invalid and excluded them from further analysis. Due to the slight variability of the scanning rate and the jitter of the telescope, the ramp-effect shapes were buried under the noise of the light curve of individual pixels. We averaged light curves of columns of pixels along the scanning direction, so that the noise caused by non-constant scanning rates was eliminated (Figure 4). Therefore, we assumed the intrinsic light curves to be a flat line for every column. We extracted 120 light curves for every visit, and we had 1800 light curves in total to fit the model parameters. The signal-to-noise ratios for the extracted light curves are approximately 1000 per exposure.

We used a Markov Chain Monte Carlo (MCMC) procedure to find the best-fit model parameters. The ramp-effect profiles are determined by 11 parameters, including the flux count rate $f$, the initial number of occupied traps $E_{0, \mathrm{~s}}$ and $E_{0, \mathrm{f}}$, the number of charge carriers captured between orbits $\Delta E_{\mathrm{s}}$ and $\Delta E_{\mathrm{f}}$, and the six parameters controlling the trapping processes $\left(E_{\mathrm{s}, \text { tot }}, \eta_{\mathrm{s}}, \tau_{\mathrm{s}}, E_{\mathrm{f}, \text { tot }}, \eta_{\mathrm{f}}, \tau_{\mathrm{f}}\right)$. We initially allowed these six parameters to vary from column to column, but found that the values of the best-fit parameters from different columns agreed with each other within uncertainties. For the rest of the study, we therefore fixed the model parameters $E_{\mathrm{s}, \text { tot }}, \eta_{\mathrm{s}}, \tau_{\mathrm{s}}$, $E_{\mathrm{f}, \text { tot }}, \eta_{\mathrm{f}}$, and $\tau_{\mathrm{f}}$ to be the same for every input light curve. In this way, we focused on the average behavior of pixels and we were able to determine these properties more precisely. The likelihood function is expressed as

$$
L=\prod_{i} \frac{1}{\sqrt{2 \pi \sigma_{i}^{2}}} \exp \left(-\frac{\left.\mathrm{obs}_{\mathrm{i}}-\operatorname{RECTE}(E, \eta, \tau, \ldots)\right]^{2}}{2 \sigma_{i}^{2}}\right) .
$$

The photometric uncertainty $\sigma$ is a combination of the photon noise, read noise, dark current, and sky subtraction noise. We assumed flat priors for $f$ and $\eta$, and flat priors in log space for $N_{0}$ and $\tau$. For $E_{0}$ and $\Delta E$, we assumed an exponential distribution as the prior distribution because, in most cases, the initially occupied traps and extra added traps are close to 0 . We 
sampled the posterior distributions and fitted 1800 light curves simultaneously so that the values of the trapping parameters $E_{\mathrm{s}, \text { tot }}, E_{\mathrm{f}, \text { tot }}, \eta_{\mathrm{s}}, \eta_{\mathrm{f}}, \tau_{\mathrm{s}}$, and $\tau_{\mathrm{f}}$ were shared among all the light curves. In order to reduce the number of free parameters, we first fitted each of the 1800 light curves to find $f, E_{\mathrm{s}, 0}, E_{\mathrm{s}, 0}$, $\Delta E_{\mathrm{s}}$, and $\Delta E_{\mathrm{f}}$. Second, we took the values of $f, E_{\mathrm{s}, 0}, E_{\mathrm{s}, 0}, \Delta E_{\mathrm{s}}$, and $\Delta E_{\mathrm{f}}$ calculated from the first step to fit for $E_{\mathrm{s}, \text { tot }}, E_{\mathrm{f}, \text { tot }}, \eta_{\mathrm{s}}$, $\eta_{\mathrm{f}}, \tau_{\mathrm{s}}$, and $\tau_{\mathrm{f}}$. We iterated the two steps and found that best-fit model parameters (Table 3) quickly converged after just five iterations.

\section{Results}

The result of the model fit is shown in Figure 5. The model parameters are well constrained. The posterior distributions of $E_{0}$ and $\tau$ are log-normal distributions, while the posterior distribution for $\eta$ is Gaussian. We calculated the best-fit values, as well as the $1 \sigma$ uncertainties for the model parameters, and list them in Table 3. The fact that the best-fit parameters are tightly constrained with small uncertainties demonstrate that our model is able to make consistent correction for every light curve in the entire data set. We note that the best-fit values for model parameters listed in Table 3 represent average values for different pixels. Although small intra-pixel variations in the trapping parameters would not be surprising, our results demonstrate that a single set of trap is able to provide high fidelity corrections for typical light curves.

The additional four parameters, $E_{0, \mathrm{~s}}, E_{0, \mathrm{f}}, \Delta E_{\mathrm{s}}$, and $\Delta E_{\mathrm{f}}$ vary on a visit to visit basis. $E_{0, \mathrm{~s}}, E_{0, \mathrm{f}}$ varies from 0 to $20 \%$ of the total numbers of traps, representing the uncertainty of the initial state of the detector. The values for $\Delta E_{\mathrm{s}}$, and $\Delta E_{\mathrm{f}}$ are normally close to zero, but help to provide finer fit early in the orbit.

We tested the validity of the model using the four-orbit-long transits of the 12 visit observations. As an example, we plotted the distributions of the model fit residuals in four orbits of a onepixel column in Figure 6. The residual distributions of the four orbits are all close to Gaussian distributions, and have standard deviations of less than 1.1 times of photon noise. We performed Shapiro-Wilk normality tests for all four-orbit observations of every one-pixel column. The residuals for four orbits of every pixel column agree with normal distributions with a $\alpha=0.1$ fidelity. We further tested the normality with quantile-quantile plots as shown in Figure 7, where we plotted the observed quantile against the quantile following Gaussian distributions. The residuals agree with straight lines for all four orbits, which further verifies that our model successfully corrected the ramp effect for all four orbits.

We further tested our model with data sets listed in Table 2 to demonstrate that our model works for observations done using different telescope and instrument configurations with different levels of incoming flux.

\subsection{Applications}

We found that our model works efficiently for a wide variety of WFC3 time-resolved observations with different instrument modes and targets.

\subsubsection{Scanning and Staring Mode Observations}

For scanning mode grism observations where the scanning direction is perpendicular to the dispersion direction, a high signal-to-noise light curve for one wavelength element can be obtained by summing up the pixel counts along the scanning direction. Random noise introduced by non-constant scanning rates and telescope jitter are mostly eliminated by this step. We directly applied RECTE to the light curve extracted from each pixel column to remove the ramp effect.

Compared to scanning mode observations, staring mode observations have two major differences for the application of RECTE. First, within a region of interest, pixels are illuminated at vastly different levels. Because the ramp-effect profile is related to the pixel count levels, the total ramp effect within the region of interest cannot be calculated as the average of pixels. Second, light curves for individual pixels may be affected by telescope jitter. With $H S T$ 's excellent pointing stability (typically less than 0.1 pixel drift per orbit), telescope jitter has little effect on the overall ramp profiles. We compared the ramp effect for light curves of simulated G141 staring mode observation images with no pointing jitter/shift and with pointing shifts of 0.1 pixel per orbit, and found that the relative difference is less than $0.01 \%$. Therefore, for staring mode and imaging observations, the ramp effect can be corrected by applying RECTE without taking pointing errors into account.

\subsubsection{Transiting Planet Example: GJ1214}

While testing the validity of the model, we also measured the transmission spectrum (Figure 8) of GJ1214b. We reached the same level of correction precision as the divide-out-of-transit method used by Kreidberg et al. (2014), and obtained a very similar transmission spectrum (see Figure 9). We note that for a few wavelength channels, the normal divide-out-of-transit method shows limitations. In Figure 10, we compared the ramp profiles provided by our model and the best-fit exponential profile in the broadband light curve. The exponential fit fails to reproduce the pattern in the light curves at the beginning of the first and second orbits, while our RECTE model matches those well. In conclusion, we find that the RECTE model matches well the accuracy of the best empirical correction yet achieved HST (taking in observations designed with the empirical correction in mind), but our observations do not rely on extra orbits to reduce the ramp effect.

GJ1214b (Charbonneau et al. 2009) is a $6.5 M_{\oplus}$ transiting planet. The large transit depth and brightness of the host star make it one of the best-suited sub-neptunes for transmission spectroscopic observations. This planet has been extensively observed both with space-based and ground-based facilities in multiple wavelengths from optical to mid-infrared (e.g., Bean et al. 2010, 2011; Fraine et al. 2013). The data that we used to constrain and validate the RECTE model were originally published by Kreidberg et al. (2014), where they found a flat near-infrared transmission spectrum for GJ1214b between 1.15 and $1.65 \mu \mathrm{m}$. The featureless transmission spectrum requires high-altitude clouds/haze in the atmosphere of GJ1214b to suppress the otherwise strong water absorption features. For their data reduction, Kreidberg et al. (2014) discarded the first orbit of every visit due to the different shapes of the rampeffect profiles. They used both empirical exponential functions and white light curves to correct the ramp effect, and obtained a consistent transmission spectrum and limb-darkening profile (Figure 9). The consistent results of this work and of Kreidberg et al. (2014) confirms that GJ1214b has a featureless nearinfrared transmission spectrum. With RECTE, future observations will not need to discard the first orbit observations, which 


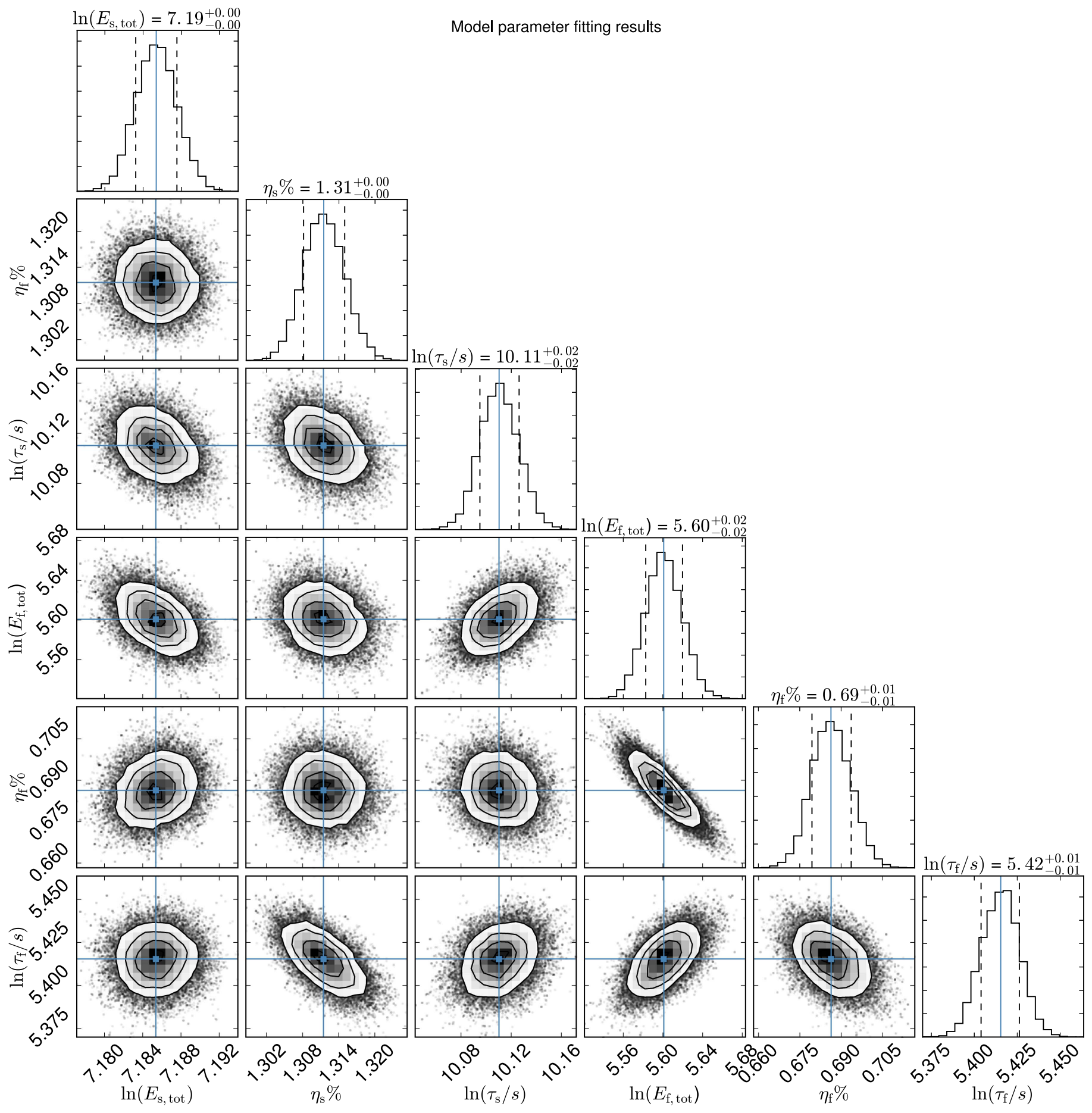

Figure 5. MCMC posterior distributions for $E_{\mathrm{s}, \text { tot }}, E_{\mathrm{f}, \text { tot }}, \eta_{\mathrm{s}}, \eta_{\mathrm{f}}, \tau_{\mathrm{s}}$, and $\tau_{\mathrm{f}}$. The blue lines mark the best-fit values while the black dashed lines show the $1 \sigma$ uncertainty. The posterior distributions of $\eta_{\mathrm{s}}$ and $\eta_{\mathrm{f}}$ are normal, while the other four are $\log$ normal. There is no significant degeneracy among these three parameters except that between $\eta_{\mathrm{f}}$ and $E_{\mathrm{f}, \text { tot }}$.

will significantly improve the efficiency of WFC 3 observations by about $25 \%$.

\subsubsection{Scanning Mode Observations: Attempting to Mitigate the Ramp with Tungsten Lamp Pre-conditioning}

Long et al. (2014) attempted to mitigate the WFC3 ramp effect by pre-conditioning the detector. They took exposures with a tungsten lamp within the instrument to fill up the traps. They turned on the lamp for $\sim 30 \mathrm{~s}$, and kept the count level for $\sim 2600 \mathrm{~s}$ to attempt to saturate the proposed charge carrier traps. The amplitudes of the ramp effect was somewhat reduced in their experiment, but they could not fully remove the ramp effect via pre-conditioning.

We applied our model to the light curves acquired in their experiment. Our model can well reproduce the ramp profiles for the detector pre-conditioned with the tungsten lamp. The results are shown in Figure 11. With our model, we found that pre-conditioning using the tungsten lamp has a very limited effect on filling up the charge carrier traps, while using the 

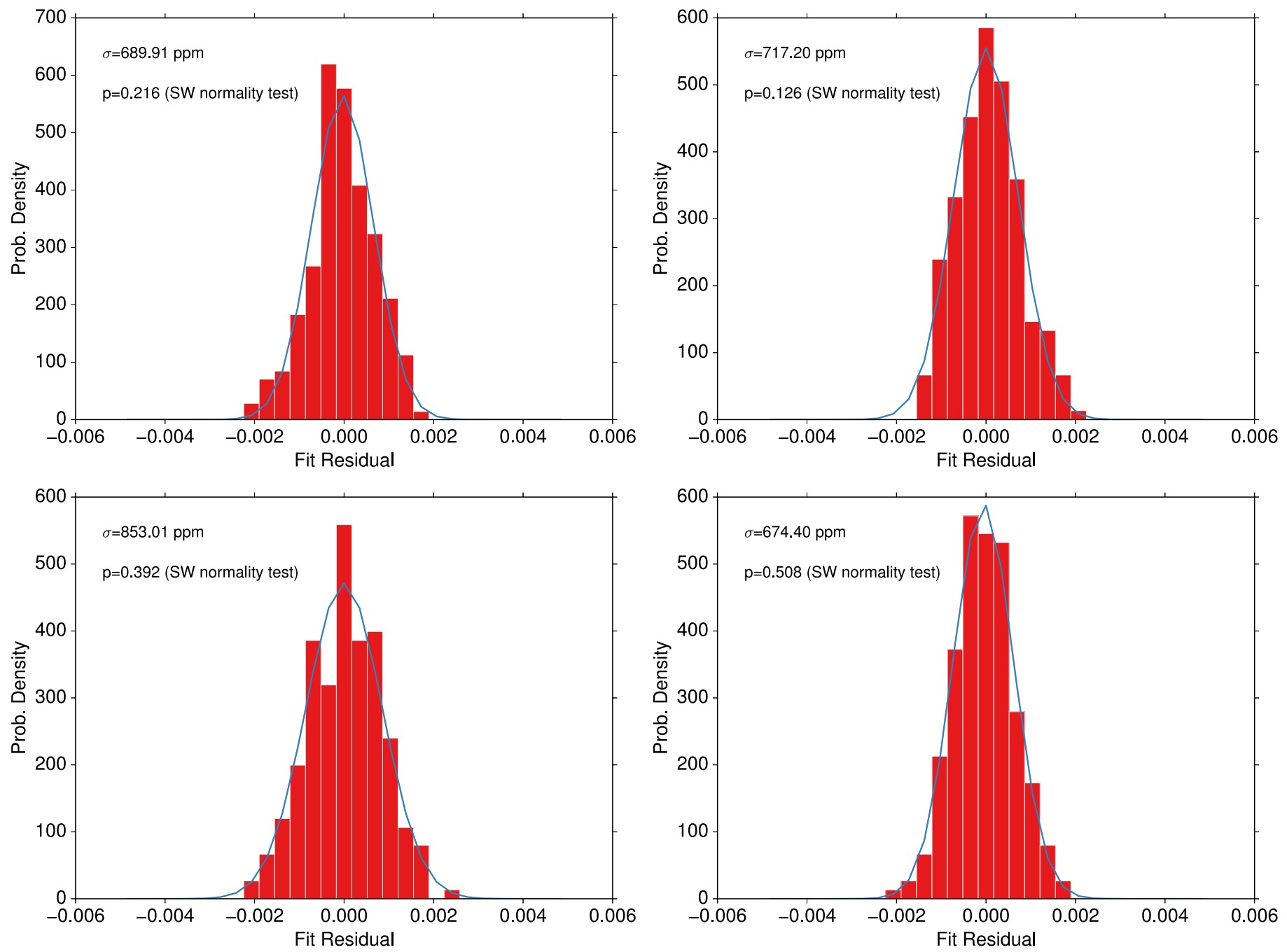

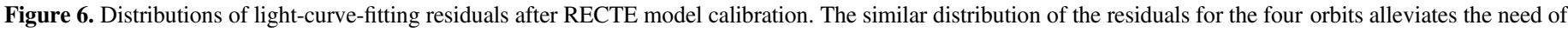
scheduling for extra orbits at the beginning of each visit for transit observations.

source itself to illuminate the detector did have a more significant effect on filling the traps. Comparing the number of filled traps after pre-conditioning with tungsten lamps with that after taking an orbit-long exposure series, we found that keeping the detector pixels at high electron levels is inefficient to fill the charge carrier traps. In the experiment of Long et al. (2014), there is one visit for which the tungsten lamp was inadvertently left on during the whole pre-condition period. The filling of the charge carrier traps appeared to be more efficient for that visit because the ramp profiles for the first and second orbits have very similar amplitudes and shapes (see Figure 3 of Long et al. 2014). However, the light curve of that visit demonstrated additional systematics that could be related to long-lasting illumination by the lamp.

\subsubsection{Rotational Phase Mapping: $2 M 0915$}

We used our model to correct WFC3 time-resolved observations of the binary brown dwarf system 2M0915 (HST program 12314, P.I. Apai) as an example to demonstrate the model's application to staring mode observations. 2M0915 system has two L7 type brown dwarfs with an angular separation of 0.77 (Reid et al. 2006). The observations included 6 orbits of G141 exposure series (SPARS25, NSAMP $=12$ exposure time
$223.73 \mathrm{~s}$ ). Each orbit had 11 exposures. For the following demonstration, we focus on the combined light curve of the binary and do not attempt to separate the two grism spectrum components. The raw light curve for the entire G141 grism wavelength span (white light curve) shows a prominent rampeffect profile in every orbit with an average amplitude of $0.5 \%$ (Figure 12), more than 10 times the photometric uncertainty.

We corrected the ramp effect with the RECTE model. The corrected light curve agrees fairly well with a flat light curve with a reduced $\chi^{2}$ of 1.76 (63 degrees of freedom). The fitting residual of straight line with the RECTE model applied has a standard deviation of $0.055 \%$ ( $1.1 \times$ the photon noise).

Rotational phase mapping from time-resolved spectroscopy provides the most direct observational constraints on the condensate clouds of ultra-cool atmospheres (e.g., Apai et al. 2013; Buenzli et al. 2015). The photometric and spectral modulations are introduced by heterogeneous clouds whose projected surface area is modulated by the rotation of the objects. The survey conducted by Buenzli et al. (2014) concluded that at least one-third of brown dwarfs with spectral types from mid-L to mid-T have observable rotational modulations in the near-IR band from 1.1 to $1.7 \mu \mathrm{m}$. Using Spitzer observations, Metchev et al. (2015) also detected rotational modulations for 30\% to $40 \%$ L3-L9.5 brown dwarfs 

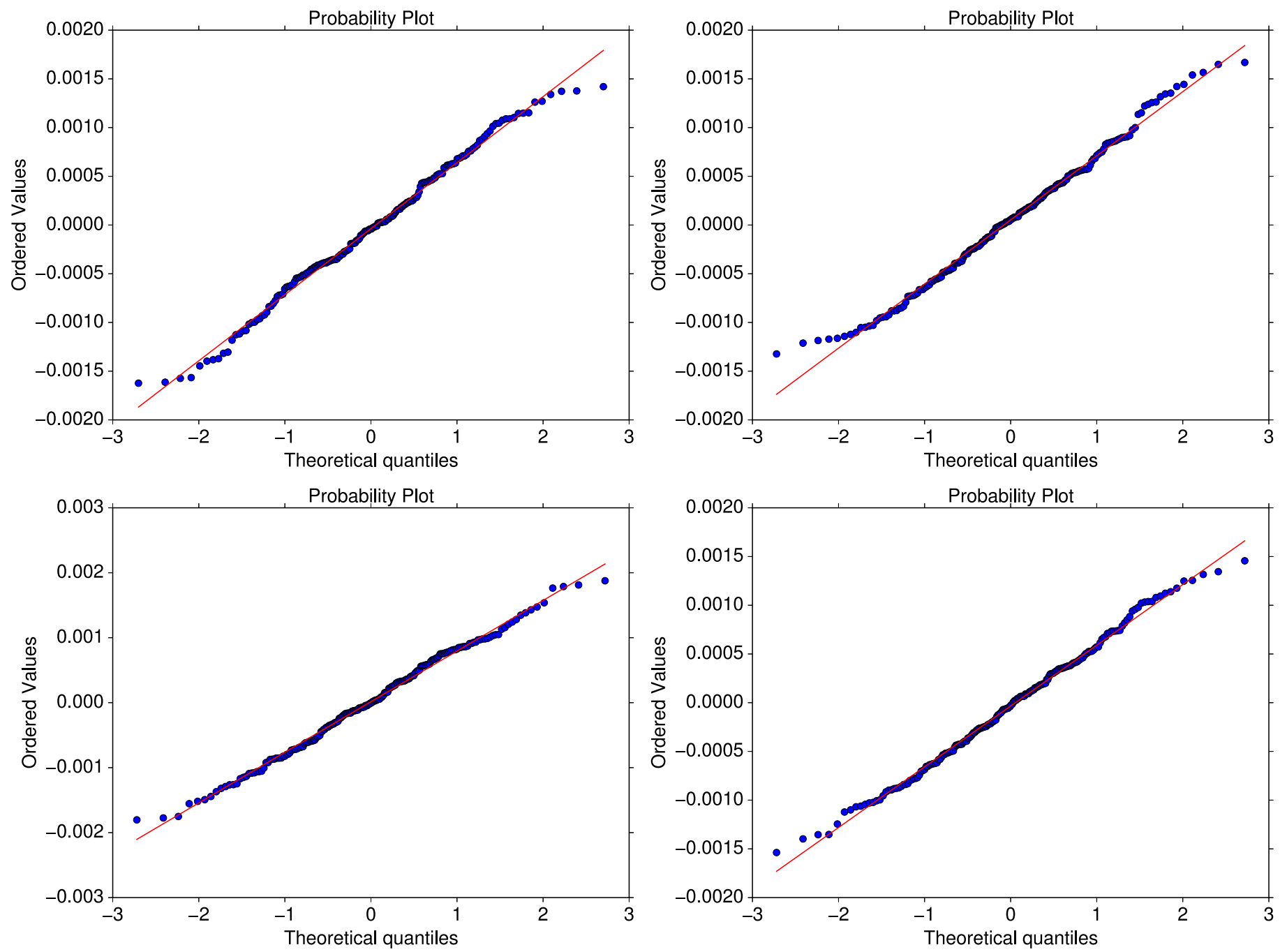

Figure 7. Quantile-quantile plot showing that the fitting residuals have very few components of red noises in all four orbits. Quantiles of the residual are plotted against the quantiles of normal distributions. Very small deviations from the linear relation demonstrate that red noises are mostly removed for all four orbits.

Table 2

WFC3 Data Used for Model Fitting and Testing

\begin{tabular}{|c|c|c|c|c|c|c|}
\hline ID & PI & No. of Orbits & Subframe & Exp. Mode & Peak Count Levels $\left(\mathrm{e}^{-}\right)$ & Observing Modes \\
\hline 12181 & Deming & 8 & 512 & RAPID, NSAMP $=16$ & $6.4 \times 10^{4}$ & staring \\
\hline 12181 & Deming & 4 & 256 & SPARS10, NSAMP $=5$ & $4.9 \times 10^{4}$ & one direction scanning \\
\hline 12251 & Berta-Thompson & 12 & 512 & RAPID, NSAMP $=7$ & $6.4 \times 10^{4}$ & staring \\
\hline 12314 & Apai & 6 & 256 & SPARS 25, NSAMP $=12$ & $1.9 \times 10^{4}$ & staring \\
\hline 13021 & Bean & 60 & 256 & SPARS10, NSAMP $=13,15$ & $2.3 \times 10^{4}$ & one/two direction scanning \\
\hline 13573 & Long & 8 & 256 & SPARS10, NSAMP $=5,6$ & $2.8 \times 10^{4} / 3.8 \times 10^{4}$ & two direction scanning \\
\hline 14241 & Apai & 28 & 256 & SPARS10, NSAMP $=5-8$ & $1.2 \times 10^{4}-3.0 \times 10^{4}$ & staring \\
\hline
\end{tabular}

in Spitzer 3.6 and $4.5 \mu \mathrm{m}$ band. They both claimed that almost all brown dwarfs have heterogeneous cloud coverages, after taking into account the random distributions of the inclinations of rotation axis and limited time coverage and sensitivity of their observations.

The corrected light curve of the brown dwarf binary 2M0915 agrees well with a flat light curve. Based on the corrected light curve, the possibility of rotational modulation with peak-topeak amplitude larger than $0.15 \%$ with a period shorter than $2.5 \mathrm{hr}$ can be excluded above the $3 \sigma$ level. The flat combined light curve of the binary brown dwarf can be interpreted as either the two binary components having very homogeneous
Table 3

RECTE Model Fit Results

\begin{tabular}{llll}
\hline \hline Parameter & Value & Parameter & Value \\
\hline$E_{\mathrm{s}, \text { tot }}$ & $1320.0 \pm 2.8$ & $E_{\mathrm{f}, \text { tot }}$ & $270.6_{-4.9}^{+5.2}$ \\
$\eta_{\mathrm{s}}$ & $1.311 \pm 0.0034 \%$ & $\eta_{\mathrm{f}}$ & $0.6863 \pm 0.0070 \%$ \\
$\tau_{\mathrm{s}}$ & $2.45_{-0.037}^{+0.0040} \times 10^{4} \mathrm{~s}$ & $\tau_{\mathrm{f}}$ & $224.8_{-2.4}^{+2.6} \mathrm{~s}$ \\
\hline
\end{tabular}

atmospheres or the spin axes of both of the two brown dwarfs having very close to pole-on inclinations. The first interpretation is unlikely, given the high occurrence rate of 


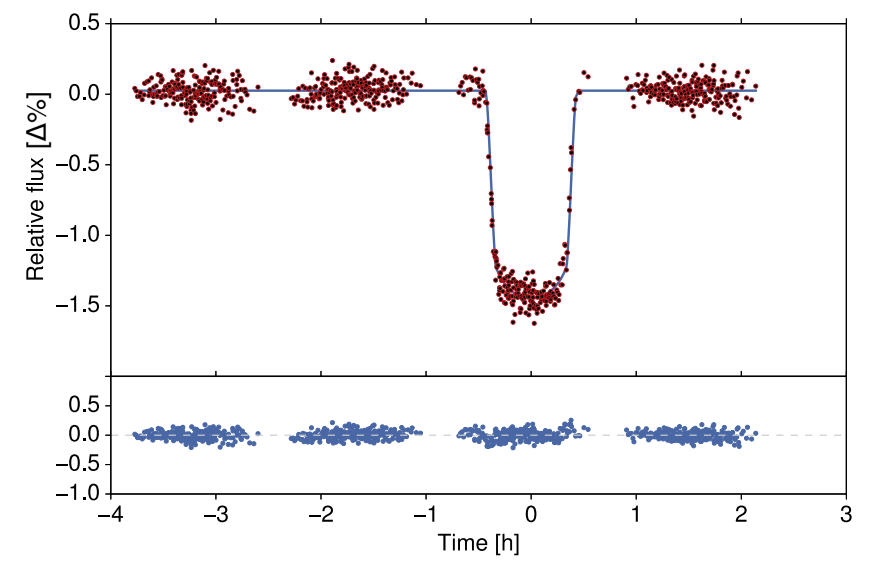

Figure 8. Four orbits of GJ1214 transit observations are well fitted by RECTE + transit profile model.

heterogeneous atmospheres. Therefore, both of the brown dwarfs should have nearly pole-on spin axes. Constraints on the orbital motion of the binary system will help study the orbit-spin alignment and the dynamical evolution in this system.

\section{Discussion}

\subsection{Mitigating the Ramp Effect}

Swain et al. (2013) proposed several methods for timeresolved observations using WFC3 to reduce the ramp effect, including using small sub-array mode and keeping low count levels $\left(<40,000 \mathrm{e}^{-}\right)$. Our model quantitatively supports some suggestions. The data storage memory of WFC3 can only save 32 full $(1014 \times 1014)$ near-IR channel frames, so high-cadence time-resolved observations with array sizes of $512 \times 512$ or above often require multiple buffer downloads within the visibility period. The buffer download time of WFC3 is 8-9 minute, which is more than five times the lifetime of the fast traps. Therefore, the fast traps will release a considerable number of charges and be available again during the buffer download time, leading to a more significant ramp effect. As a comparison, the buffer download times for $256 \times 256$ and $128 \times 128$ sub-arrays are similar to or even shorter than the fast trap lifetime. Observations taken in these two small subarray modes normally do not require buffer downloads in the middle of the orbits. Therefore, there are no significant breaks in the exposure series during which trapped charge carriers can be released but not added during orbits when the smaller subarrays are used. As for the recommendation for keeping low count levels, we see no evidence for the need to calibrate the ramp effect differently for different exposure levels (i.e., the same trap parameters provide excellent fits). Observations with relatively low count levels (e.g., 2M0915) and high count levels (e.g., HD 209458) have similar ramp-effect amplitudes with different apparent ramp-effect profiles, and can be corrected by the same model.

When applying RECTE to correct the ramp effect, the only degrees of freedom of the ramp profiles are the initial numbers of the charge carrier traps, and the extra numbers of charge carriers trapped during the gaps between the orbits. The degrees of freedom of the model can be further decreased to acquire more accurate ramp-effect correction, if the initial states and telescope pointing information between the orbits can be recorded. Therefore, for future observations, we suggest that detector images should be recorded before the telescope is reset prior to the science observations to allow for the measurement of the illumination levels the detector was exposed to during the inter-orbit gaps. Alternatively, or in addition, the detector's inter-orbit exposure should be minimized during high-precision time-resolved observations: because the WFC3/IR detector has no mechanical shutter, we recommend that the filter wheel be set to a "blank" (opaque blocker) position, also used to prevent the detector from viewing Earth during occultations.

\subsection{The Relationship between the Number of Traps and Fluence Levels}

The theories of charge carrier trapping (e.g., Smith et al. 2008a) suggest that the total number of charge carrier traps can increase if the illumination fluence levels are very high. The width of the undepleted regions (see Figure 1 of Smith et al. 2008a) increases with fluence level, which potentially enlarges the number of available traps. Indeed, Long et al. (2012, 2015a) showed that the persistence of the WFC3 IR detector surged when fluence reached near saturation, which could suggest significant trap density increase at saturation. However, for the scientific cases that this study focuses on, in which the maximum fluence levels are almost always kept well below the saturation, we see no evidence for this effect, and a constant level of available traps reproduces the observations very well.

Figure 1 demonstrates a qualitative trend by which the first orbit light curve ascends faster with increasing count level and flux. For example, from Figure 1 panels A to D, as the flux gets lower, the slope of the first five points also decreases $(0.10$ [\%/min], $0.088[\% / \mathrm{min}], 0.046[\% / \mathrm{min}]$, and $0.034[\% / \mathrm{min}]$, respectively). If we assume that the number of traps are proportional to the fluence level, given a fixed exposure time, the exponential index in Equation 8 and the ramp profiles would be independent of illumination flux, which contradicts the observed trend. To illustrate this point, we compare the first two orbits of the scanning mode observation of HD 209458 (Figure 1(A), a transiting hot-Jupiter host; Deming et al. 2013) to two-model light curves as shown in Figure 12. Note that the average fluence level for HD 209458 (Figure 1(A)) is more than twice that of GJ1214 (Figure 1(B)). In the first model, we use the same model parameters as the best-fit parameters for GJ1214. In the second model, we proportionally increased the number of traps for both populations based on the different fluence levels of two observations. As shown in Figure 13, the second model cannot fit the steep ramp in the first orbit as well as the first model does, resulting in fitting residuals twice as large as those from the first model. Therefore, we conclude that for observations with fluence levels well below saturation, there is no benefit from considering varying trap numbers for rampeffect calibrations.

\subsection{Ramp Effect with Image Persistence in WFC3 IR}

The idea of charge carrier trapping originated from studies of image persistence. A complete model of charge carrier trapping should naturally explain both the ramp effect and image persistence. From an empirical perspective, a series of studies by Long et al. (2012, 2015a, 2015b) provided the most accurate model to predict image persistence for WFC3 IR detectors yet. This model is publicly available as the "persistence pipeline." 

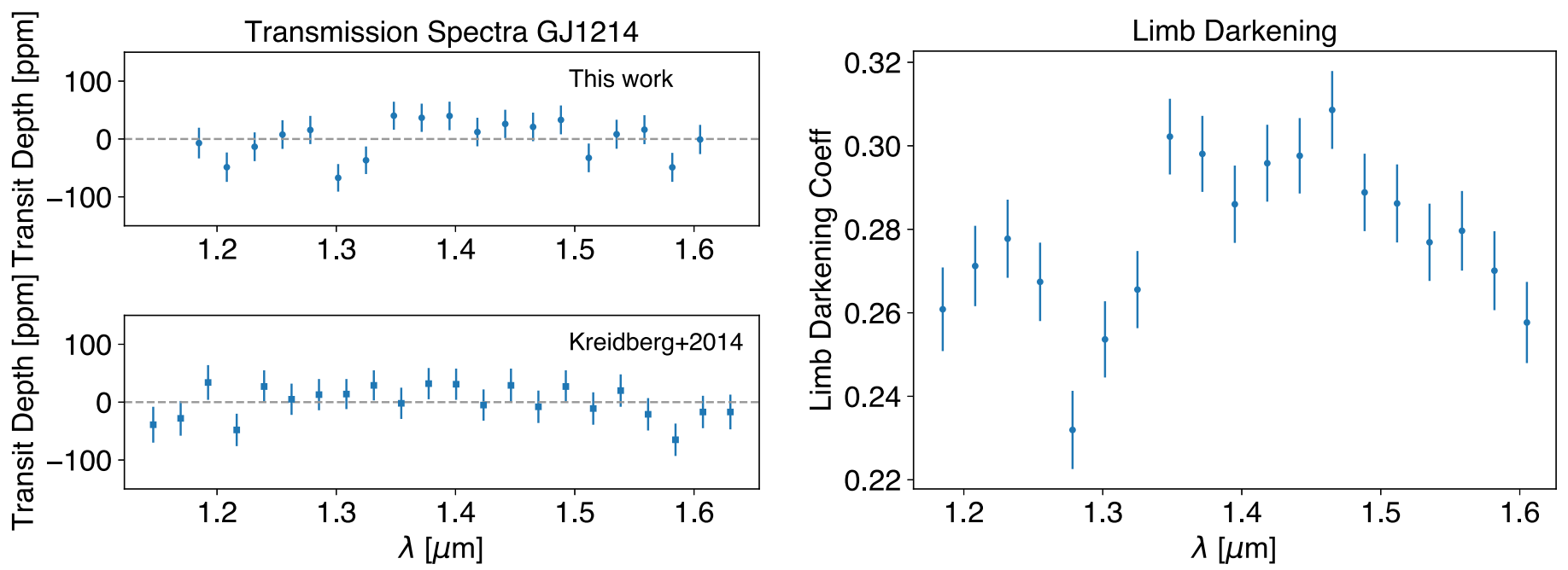

Figure 9. Transmission spectra (left) and limb-darkening coeffecient (right) measured from this work and a comparion to those of Kreidberg et al. (2014). The measurements agree well within uncertainties.

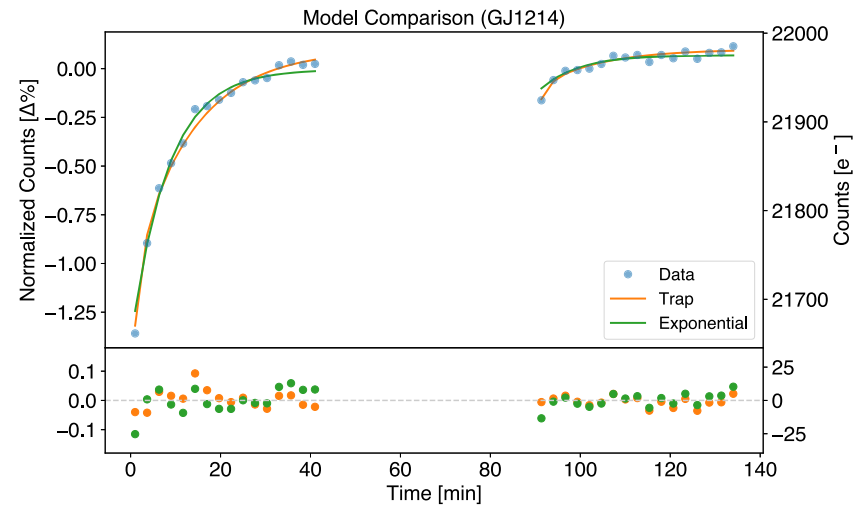

Figure 10. Comparison of systematics corrections and fitting residuals for one light curve from the GJ1214 data set. The orange curves and dots are ramp profiles calculated using the proposed model and its fitting residuals, and the green curves and dots are those for the best exponential fit using the white light curves. Because of the change of ramp profiles across different wavelengths, the best-fit exponential profile failed to fit the beginning of both orbits.

The key characteristics of the model of Long et al. include a significant rise of persistence for fluence near-saturation levels, persistence decaying as a power law, and persistence being related to the exposure times. In contrast, the model presented here does predict that persistence varies with exposure time, but saturation is not relevant in our model, and the persistence decays exponentially after the end of the exposures. For fluence below $50000 \mathrm{e}^{-}(2 / 3$ of the saturation level), our model predicts persistence of less than $0.05 \mathrm{e}^{-}$after $500 \mathrm{~s}$ of the exposure, which is at an order similar to the observational measurements of Long et al. (2012, 2015a).

The differences between the two models reflect their different goals: our model was developed to correct the ramp effect in high-cadence, short exposure time observations, while the model by Long et al. corrects for persistence in exposures that follow long integrations and fluence levels close to saturation. For the observations that our model focuses on, the trapping of the charge carriers normally reduces the observed flux by $\sim 0.2 \%-1.5 \%$, while the increase of the observed flux from persistence (released charge carriers) is two orders of magnitude lower. The persistence profile in Long et al. $(2012,2015 a)$ can be qualitatively explained with a third population of traps that has a broad range of trapping lifetimes that are only activated when fluence is above a certain threshold. Combining the approaches by Long et al. for the persistence with our model for the ramp effect should be possible and would result in a powerful, general, and broadly applicable model for charge trapping in WFC3/IR, but it is beyond the scope of our study.

\subsection{The Extension of RECTE to Other Detectors}

Because many IR detectors used in astronomy are manufactured using the similar technology as WFC3, in principle, RECTE can be extended to other detectors by adjusting the model parameters. Specifically, the two nearinfrared instruments on board the James Webb Space Telescope (JWST), NIRCam, and NIRSpec have very similar focal plane arrays as those of WFC3, and produced by the same manufacturer (Teledyne Scientific \& Imaging). NIRCam has two identical branches, each of which contains two $2 \mathrm{k} \times 2 \mathrm{k}$ $\mathrm{HgCdTe}$ arrays that cover the wavelength ranges of $0.6-2.4 \mu \mathrm{m}$ and 2.4-5.0 $\mu \mathrm{m}$ (Burriesci 2005). The focal plane array of NIRSpec consists of two butted HgCdTe HAWAII-2RG sensor chip assemblies (Bagnasco et al. 2007). The extension of our model to NIRCam may improve the accuracy of time-resolved observations and save the valuable JWST time by alleviating the need to wait for the detector state to "settle."

With its larger photon collecting area and higher sensitivity compared to WFC3, JWST instruments create new opportunities for exoplanet atmosphere studies, and are expected to devote large fractions of available time for time domain exoplanet observations. For example, with NIRCam, it is expected that with a single transit observation, a signal-to-noise level of 55 at a spectral resolution of $R \sim 55$ can be achieved (Beichman et al. 2014). With such high-quality observations, faint spectral features may be visible even for GJ1214b, where high-altitude hazes suppress spectral features. However, the performance of JWST may be limited by an inadequate understanding of the systematics effect, and it is likely that $J W S T$ NIRCAM may also show a similar ramp effect. In that case, The extension of RECTE may be used to solve potential ramp-effect systematics for JWST/NIRCam and JWST/NIRSpec to obtain high accuracy and efficiency observations. 

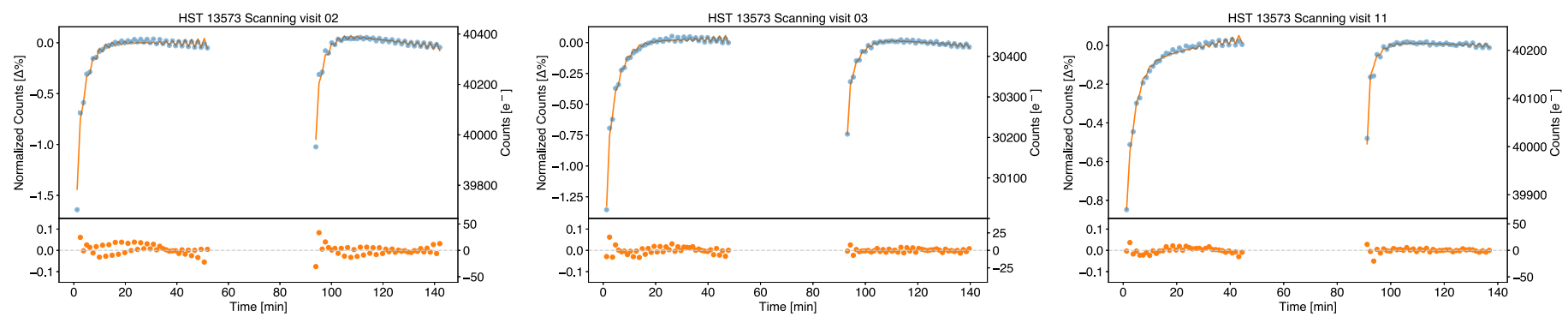

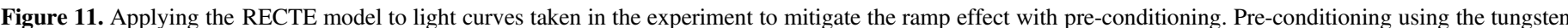

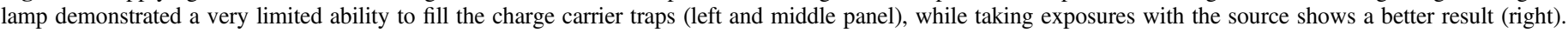

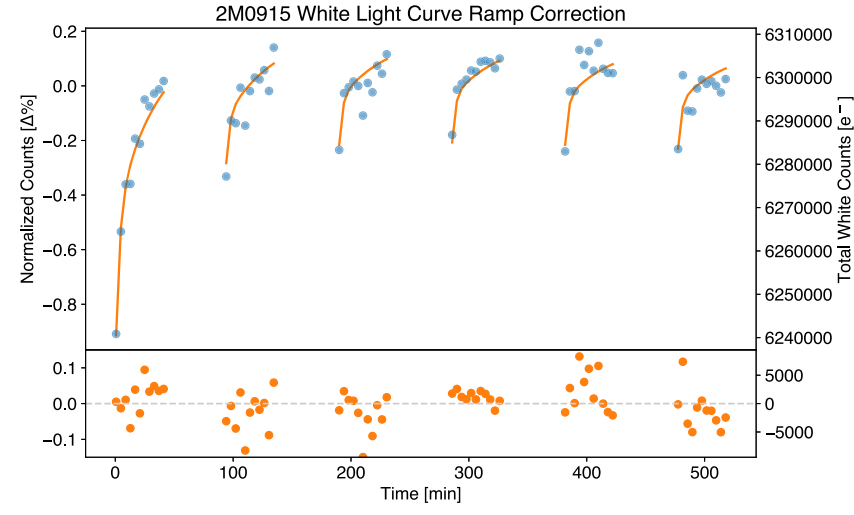

Figure 12. Six-orbit observations of binary brown dwarf binary 2M0915 corrected by RECTE.

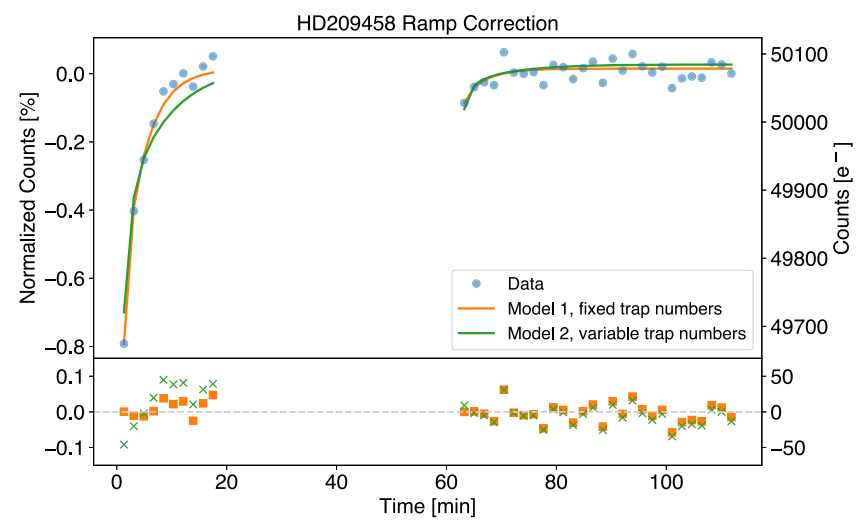

Figure 13. Light curve of the first two orbits of the scanning mode observations of transiting exoplanet HD209458b compared with two-model curves. Despite the different incoming flux compared to GJ1214b, the rampeffect trend can be corrected with high accuracy with model parameters exactly the same as those fitted using the scanning mode observations of GJ1214b. In contrast, the model (green) that assumes that the number of traps is directly proportional to the fluence level predicts a light curve that is inconsistent with the observations.

\section{Summary}

We present a physically motivated model RECTE to correct the WFC3 ramp effect, the most significant systematic effect in time-resolved observations with WFC3. We created the model based on the theory of charge carrier trapping in $\mathrm{HgCdTe}$ arrays, and quantitatively constrained and tested our model by using more than 120 orbits of archival WFC3 time-resolved observations. Our model enables the high fidelity correction of the WFC3 ramp effect for observations taken in both staring and scanning modes regardless of the instrument setup. Light curves corrected by RECTE have their residual photometric systematic errors reduced by a factor of 10 , and are nearly photon noise limited.

Our model can be easily implemented in existing light-curve analysis pipelines. We demonstrate the use of RECTE on two astrophysically relevant examples and discuss the results of the observations. The two examples are transiting exoplanet spectroscopy, and brown dwarf rotational phase mapping, the two most commonly used time-resolved observational techniques with WFC3.

Existing and future time-resolved observations with WFC3 will benefit from our model and will provide better systematics correction and improve on-orbit observing efficiency.

1. Our model does not require a flat baseline to separate the instrument-related variability from the variability that is intrinsic to the target. Therefore, compared to data-driven methods that cannot separate instrumental and astrophysical signals, our model provides superior ramp-effect correction for observations for which flat baselines are not available. Observations of transiting exoplanet phase curves and direct photometric/spectroscopic rotational phase mapping immediately benefit from our model.

2. For uneven illuminations, e.g., a strong absorbing feature in part of the spectral image, different count levels lead to different ramp profiles. In the empirical correction, the pixels that are used to calculate the correction terms do not have the same count levels as the pixels that are corrected. For example, in transit spectroscopy, the correction term obtained from white light curves may not accurately correct the ramp profile in wavelength channels where the illumination level is different from the average level of the white light curve. Our model provides a more accurate correction, especially in cases where the planet-hosting stars have significant spectral features within the wavelength range of the observations.

3. Our model corrects the ramp effect in the first orbit of each $H S T$ visit just as well as in the rest of the orbits. In future observations, the first orbit light curves no longer have to be excluded from the analysis. For the most used transit observation configuration of four continuous orbits per transit, our model will increase the $H S T$ observing efficiency by $25 \%$ by alleviating the need of discarding the first orbit.

4. Our model can be easily extended to similar detectors if a similar effect is observed. The extensions of our model to NIRCam/JWST and NIRSpec/JWST are expected to improve the accuracy and efficiency of future JWST observations. 
We would like to thank the referee Knox Long for providing a thorough report, which led to the considerable improvement of the manuscript. We would like to thank Peter McCullough for a discussion on WFC3 detectors and comments on the manuscript, Tom Evans, David Sing, Diana Dragomir, and Hannah Wakeford for helpful discussions on WFC3 transiting exoplanet observations, Laura Kreidberg for clarifications of GJ1214 data reduction, Marcia Rieke for a discussion on JWST/NIRCam, and Carmen Ortiz Henley for editorial help. Y.Z. is supported in part by the NASA Earth and Space Science Fellowship Program-Grant "NNX16AP54H," and the Technology Research Initiative Fund (TRIF) Imaging Fellowship, University of Arizona. D.A. acknowledges support by the National Aeronautics and Space Administration under agreement No. NNX15AD94G for the program Earths in Other Solar Systems. B.W.P.L. is supported in part by the Technology Research Initiative Fund (TRIF) Imaging Fellowship, University of Arizona. Support for program number 12314, 13418, and 14241 were provided by NASA through a grant from the Space Telescope Science Institute, which is operated by the Association of Universities for Research in Astronomy, Inc., under NASA contract NAS5-26555.

Software: Numpy\&Scipy (van der Walt et al. 2011), Matplotlib (Hunter 2007), IPython (Perez \& Granger 2007), Astropy (Robitaille et al. 2013), emcee (Foreman-Mackey et al. 2013), corner.py (Foreman-Mackey 2016).

\section{References}

Agol, E., Cowan, N. B., Knutson, H. A., et al. 2010, ApJ, 721, 1861 Anderson, R. E., Regan, M., Valenti, J., \& Bergeron, E. 2014, arXiv: 1402.4181

Apai, D., Radigan, J., Buenzli, E., et al. 2013, ApJ, 768, 121

Baggett, S., Hill, R., Kimble, R., et al. 2008, Proc. SPIE, 7021, 70211 Q

Bagnasco, G., Kolm, M., Ferruit, P., et al. 2007, Proc. SPIE, 6692, 66920M

Bean, J. L., Désert, J.-M., Kabath, P., et al. 2011, ApJ, 743, 92

Bean, J. L., Miller-Ricci Kempton, E., \& Homeier, D. 2010, Natur, 468, 669

Beichman, C., Benneke, B., Knutson, H., et al. 2014, PASP, 126, 1134
Berta, Z. K., Charbonneau, D., Désert, J.-M., et al. 2012, ApJ, 747, 35 Buenzli, E., Apai, D., Morley, C. V., et al. 2012, ApJL, 760, L31 Buenzli, E., Apai, D., Radigan, J., Reid, I. N., \& Flateau, D. 2014, ApJ, 782, 77 Buenzli, E., Saumon, D., Marley, M. S., et al. 2015, ApJ, 798, 127

Burriesci, L. G. 2005, Proc. SPIE, 5904, 21

Charbonneau, D., Berta, Z. K., Irwin, J., et al. 2009, Natur, 462, 891

Deming, D., Wilkins, A., McCullough, P., et al. 2013, ApJ, 774, 95

Dressel, L. 2016, Wide Field Camera 3, HST Instrument Handbook, 1

Foreman-Mackey, D. 2016, The Journal of Open Source Software, 24 doi:10. 5281 /zenodo.45906

Foreman-Mackey, D., Hogg, D. W., Lang, D., \& Goodman, J. 2013, PASP, 125,306

Fraine, J. D., Deming, D., Gillon, M., et al. 2013, ApJ, 765, 127

Hunter, J. D. 2007, CSE, 9, 90

Kreidberg, L., Bean, J. L., Désert, J.-M., et al. 2014, Natur, 505, 69

Lew, B. W. P., Apai, D., Zhou, Y., et al. 2016, ApJL, 829, L32

Long, K. S., Baggett, S. M., \& Mackenty, J. W. 2015a, STScI Instrument Science Report WFC3, 15

Long, K. S., Baggett, S. M., \& Mackenty, J. W. 2015b, STScI Instrument Science Report WFC3, 16

Long, K. S., Baggett, S. M., MacKenty, J. W., \& McCullough, P. M. 2014, STScI Instrument Science Report WFC3, 14

Long, K. S., Baggett, S. M., MacKenty, J. W., \& Riess, A. G. 2012, Proc. SPIE, 8442, 84421W

McCullough, P., \& MacKenty, J. 2012, STScI Instrument Science Report WFC3, 8

Metchev, S. A., Heinze, A., Apai, D., et al. 2015, ApJ, 799, 154

Perez, F., \& Granger, B. E. 2007, CSE, 9, 21

Reid, I. N., Lewitus, E., Allen, P. R., Cruz, K. L., \& Burgasser, A. J. 2006, AJ, 132, 891

Rieke, G. H. 2012, Measuring the Universe (Cambridge: Cambridge Univ. Press)

Robitaille, T. P., Tollerud, E. J., Greenfield, P., et al. 2013, A\&A, 558, A33

Smith, R. M., Zavodny, M., Rahmer, G., \& Bonati, M. 2008a, Proc. SPIE, 7021, 70210J

Smith, R. M., Zavodny, M., Rahmer, G., \& Bonati, M. 2008b, Proc. SPIE, $7021,70210 \mathrm{~K}$

Swain, M., Deroo, P., Tinetti, G., et al. 2013, Icar, 225, 432

van der Walt, S., Colbert, S. C., \& Varoquaux, G. 2011, CSE, 13, 22

Varley, R., Tsiaras, A., \& Karpouzas, K. 2015, arXiv:1511.09108

Wakeford, H. R., Sing, D. K., Evans, T., Deming, D., \& Mandell, A. 2016, ApJ, 819, 10

Zhou, Y., Apai, D., Schneider, G. H., Marley, M. S., \& Showman, A. P. 2016, ApJ, 818, 176 